\title{
TITLE: GPIa/GPIIa GENE POLYMORPHISM AND BLEEDING AFTER CARDIAC SURGERY
}

AUTHORS: Natasha Kraeva PhD, George Djaiani MD, Vyacheslav Morosov PhD, Mark Busato, Jo Carroll RN, Bobbi Jo Anderson, Ludwik Fedorko MD

AFILLIATION: Department of Anesthesia \& Pain Management, Toronto General Hospital, UHN, Banting \& Best Dept. of Medical Research, UofT, 200 Elizabeth Street, Toronto, ON, M5G 2C4

INTRODUCTION: Adverse hematological outcome in cardiac surgical patients is defined by interplay of environmental and genetic factors. Among genetic factors common polymorphisms in the genes encoding haemostatic factors have been recently shown to influence their plasma levels. These polymorphisms have been extensively studied in relation to pro-thrombotic states, but their role in surgical blood loss is still unknown. Factor V Leiden has been reported to reduce blood loss after cardiac surgery ${ }^{1}$. Here we report preliminary results of a pilot prospective case control study on identification of risk factors predictive of excessive perioperative bleeding in cardiac surgical patients.

METHODS: Following REB approval patients were stratified into two groups: bleeders with mean blood loss $1166 \mathrm{ml}$ (95\%CI; 942.08- 1389.9) during first 12 hours after surgery and non-bleeders with blood loss $433 \mathrm{ml}$ (95\% CI; 394,8 - 471. 9). Seven common polymorphisms known to affect hemostatic cascade were selected for our study (table). Genotyping was performed by RFLP analysis and direct sequencing. Allele frequencies were estimated by gene counting. Hardy-Weinberg equilibrium was tested by $\mathrm{chi}^{2}$ analysis with $1 \mathrm{df}$. Differences in the allele frequencies between patient groups were compared with the $\mathrm{chi}^{2}$ - test.

RESULTS: Genotype distributions in bleeders $(2 n=20)$ and nonbleeders $(2 n=162)$ were in HardyWeinberg equilibrium. Frequencies of minor alleles in two study groups and in control populations are summarized below. FV Leiden statistical analysis was not feasible due to the low frequency (0.02) and small sample size.

\begin{tabular}{|l|l|l|l|l|l|l|}
\hline Gene & Allele & Bleeders & Non bleeders & P value & French $^{2}$ & Spanish $^{2}$ \\
\hline GPIa & C807T & 0.15 & 0.39 & $\mathrm{P}<0.025$ & $0.4^{3}$ & \\
GPIIIa & Leu33Pro & 0.3 & 0.172 & NS & 0.143 & 0.1611 \\
F2R & (-506)I/D & 0.25 & 0.24 & NS & $0.247^{4}$ & \\
Fibrinogen & G(-455)A & 0.15 & 0.15 & NS & 0.195 & 0.222 \\
Factor FVII & (-323)I/D & 0.1 & 0.12 & NS & 0.18 & 0.16 \\
Factor FVII & Arg353Gln & 0.1 & 0.12 & NS & 0.18 & 0.16 \\
\hline
\end{tabular}

DISCUSSION Most of studied allele frequencies were not significantly different between the two groups and similar to the reported frequencies for these polymorphisms for Caucasian populations. In contrast, the C807 allele of the GPIa was significantly more frequent among bleeders than nonbleeders indicating its relevance as a risk factor for surgical bleeding. The C807 allele was shown to be associated with lower efficiency of platelet adhesion ${ }^{3}$. This might contribute to an increased risk of surgical bleeding. However, a larger patient cohort is required to confirm or reject these findings.

\section{REFERENCES}

1. Circulation. 107:1003-8; 2.Genomics 83: 559-5652; 3. Arterioscler Thromb Vasc Biol. 22: 14-203;

4. Arterioscler Thromb Vasc Biol. 20: 585-592 